

\title{
Cooper Pair Transport in a Resistor-Biased Josephson Junction Array
}

\author{
Sergey V. Lotkhov, Vladimir A. Krupenin, and Alexander B. Zorin
}

\begin{abstract}
The dc transport properties of long arrays of small Al Josephson junctions, biased through on-chip $\mathrm{Cr}$ resistors, are studied. The $I V$-characteristics show a large Coulomb threshold for current as well as negative-slope regions indicating the regime of autonomous Bloch oscillations up to rather high frequencies of $f=I / 2 e \sim 1 \mathrm{GHz}$, comparable to those reported by other groups for single junctions. On the other hand, a small depth of the back-bending implies a low duty cycle and a broad spectrum of the oscillations, which we attribute to the insufficiently high impedance of the bias resistors. A self-sustained switching process at a small bias current is used to study the statistics of the switching voltages and to determine the effective Bloch capacitance which was found to considerably exceed the geometric junction capacitance.
\end{abstract}

\section{Index Terms}

Charge transfer, current, Josephson arrays, SQUIDs, superconductor-insulator-superconductor devices, thin-film devices, stripline components.

\section{INTRODUCTION}

The application of single-charge tunneling effects in electrical metrology has been considered in a view of capacitance and current standards based on charge quantization phenomena (see, e.g., review [1]). The proper charge quantization in circuits with small tunnel junctions has been achieved for low junction transparencies: $R_{\mathrm{T}} \gg R_{\mathrm{Q}} \equiv h / e^{2} \approx 25.8 \mathrm{k} \Omega$ for the single-electron

Manuscript received July 10, 2006. This work was partially supported by the European Commission within the project EuroSQIP.

S. V. Lotkhov and A. B. Zorin are with Physikalisch-Technische Bundesanstalt, D-38116 Braunschweig, Germany.

V. A. Krupenin is with Laboratory of Cryoelectronics, Moscow State University, 119899 Moscow, Russian Federation. 
tunnelling resistance, and $E_{\mathrm{J}} \ll E_{\mathrm{C}}$ for Cooper pair devices, with $E_{\mathrm{J}} \propto R_{\mathrm{T}}^{-1}$ and $E_{\mathrm{C}}$ being the Josephson coupling and charging energies, respectively. For example, remarkable advances have been made in development towards the single-electron standard of capacitance [2]. The accurate operation of these circuits, based on pumping of single electrons, has been, however, limited to small currents, $I \sim 1$ to $10 \mathrm{pA}$, because of the stochastic nature of single electron tunneling with a relatively long time constant.

Here we address the Cooper pair transport in the case of substantial Josephson coupling $E_{\mathrm{J}} \geq$ $E_{\mathrm{C}}$, i.e., when both the Josephson phase and the charge exhibit significant quantum uncertainties. In such rather transparent, and therefore large-current-capable junctions, high accuracy could still be achieved due to the exact $2 e$-periodicity of their energy bands as a function of the quasicharge $[3]$.

\section{BASIC IDEA AND SAMPLE}

The related transport phenomenon is known as Bloch oscillations in a current-biased junction with a fundamental current-frequency relation $I=2 e f$ [3]. Successful attempts to phase-lock the Bloch oscillation by an external signal were made in the early 1990s (see, e.g., [4], [5], [6]) in the single junctions biased through high-ohmic on-chip microresistors for frequencies up to $f \sim 10 \mathrm{GHz}$. Unfortunately, the linewidth of oscillations was found to be rather large, $\delta f \sim 1 \mathrm{GHz}$, which was supposedly due to significant thermal fluctuations which smeared the current plateaus. Moreover, a possible size of these plateaus was expected to be rather small, scaling as a small threshold voltage in a single junction.

Our approach is based on 1D arrays of small Josephson junctions. As was shown theoretically for current-biased arrays [7] and proved experimentally for very long voltage-biased Al arrays $(N \sim 200)$ [8], these arrays are analogous to long Josephson junctions: Similar to the fluxons formed in the long junctions, the charge profile in the array is governed by the sine-Gordon type of equation, describing the Cooper pair solitons of size (in number of junctions) $M \sim \sqrt{\tilde{C} / C_{0}}<$ $N$ [8]. Here we denote as $\tilde{C}$ the effective junction capacitance, related to the curvature of the ground state energy $E(q)$ and, hence, the shape of this $2 e$-soliton; $C_{0} \ll \tilde{C}$ is the self-capacitance of the intermediate islands. The coherent motion of a train of $2 e$-solitons along an array biased via high-ohmic resistors can be described by a set of non-stationary equations: 


$$
L_{B}\left(q_{i}\right) \frac{\partial^{2} q_{i}}{\partial t^{2}}+\frac{\partial E\left(q_{i}\right)}{\partial q_{i}}=\frac{1}{C_{0}}\left(q_{i-1}-2 q_{i}+q_{i+1}\right), \quad i=1,2, \ldots
$$

which includes the quasicharge-dependent Bloch inductances of the junctions $L_{\mathrm{B}}\left(q_{\mathrm{i}}\right)$ which were recently introduced in [9], with corresponding initial and boundary conditions. Here we denote the set of junction quasicharges as $\left\{q_{i}(t)\right\}$. In the limit of small Josephson coupling, $\lambda \equiv$ $E_{\mathrm{J}} / E_{\mathrm{C}} \ll 1$, the periodic term $\partial E\left(q_{i}\right) / \partial q_{i}$ takes the form of the sawtooth function with the amplitude $e / C$, where $C$ is the capacitance of the individual junctions in the array. In the opposite case, $\lambda \gg 1$, there is an analytic expression [3]:

$$
\frac{\partial E\left(q_{i}\right)}{\partial q_{i}}=a(\lambda) \frac{e}{C} \sin \left(\pi q_{i} / e\right)
$$

where $a(\lambda)=2^{11 / 4} \pi^{1 / 2} \lambda^{3 / 4} \exp \left[-(8 \lambda)^{1 / 2}\right]$ is a numerical factor describing exponential suppression of the Bloch band width for $\lambda \rightarrow \infty$.

In this paper, we report the dc properties of autonomous arrays of $N=60$ SQUIDs (see the SEM photo in Fig. 1), fabricated using the Al/oxide/Al-Cr shadow evaporation process (see, e.g., [10]) in a 4-point layout with $\mathrm{Cr}$ microstrips of $50 \mu \mathrm{m}$-length with $R_{\mathrm{Cr}} \approx 550 \mathrm{k} \Omega$ each. The tunnel resistance was $R_{\mathrm{T}} \approx 8 \mathrm{k} \Omega$ and an estimated capacitance was $C_{\mathrm{T}} \sim 0.36 \mathrm{fF}$ per junction, which corresponds to the maximum Josephson coupling energy of each SQUID, $E_{\mathrm{J}} \approx 160 \mu \mathrm{eV}$ and the charging energy $E_{\mathrm{C}} \equiv e^{2} / 4 C_{\mathrm{T}} \approx 110 \mu \mathrm{eV}$ per link, yielding their ratio $\lambda \approx 1.5$. We roughly estimate $C_{0} \sim 50 \mathrm{aF}$.

The samples were measured in the current-bias mode with an external high-ohmic bias resistor, $R_{\mathrm{B}}=100 \mathrm{M} \Omega$. The capacitance of each connection line to ground was $C_{\mathrm{L}} \sim 1 \mathrm{nF}$, resulting in an $R C$-constant of the bias circuitry of $\tau_{\mathrm{D}} \sim R_{\mathrm{B}} C_{\mathrm{L}}=0.1 \mathrm{~s}$. Our microwave-tight holder was supplied with Thermocoax ${ }^{\circledR}$ filters (jacket $\varnothing 0.35 \mathrm{~mm}, 110 \mathrm{~cm}$ long), anchored to the mixing chamber.

\section{Results}

Several important peculiarities can be observed in the $I V$-characteristics of the arrays shown in Fig. 2. In our opinion, the most remarkable transport property of the system relates to the bias current range $I_{\text {bias }} \leq 300$ to $350 \mathrm{pA}$, with zero to negative slope of the $I V$-curve (see the blow-up of the corresponding region in the right inset in Fig. 2), which we assume to 
be an indication of the Bloch oscillation regime [3]. Due to the moderate current values, the ramping of quasicharge in this transport regime occurs sufficiently slowly that the system always stays in the zero Bloch band and experiences 2e-periodic oscillations of the voltage with the frequencies up to $f=I_{\text {bias }} / 2 e \sim 1 \mathrm{GHz}$. At larger currents, the average voltage across the array gradually increases, presumably due to excitation of the upper energy bands [3], thus setting a high-frequency limit for possible application of these particular Josephson arrays as sources of a quantized current. Note that, despite a large number of junctions in our arrays, this limitation, being at the level of $\mathrm{GHz}$, is virtually of the same scale with the frequencies of phase-locking experiments on single junctions [4], whereas the typical voltage scale is much larger, which is promising for a better observability of the Bloch oscillations.

On the other hand, small depth of the back-bending, typically, few percent, points out the low duty cycle of Bloch oscillations with the short pulse duration of $\tau_{\text {arr }} \leq R_{\mathrm{Cr}} C_{\text {arr }} \sim(5 \mathrm{GHz})^{-1} \ll$ $f^{-1}$, where we use a rough estimate for the "input" capacitance of the array, $C_{\text {arr }} \approx\left(2 \tilde{C} C_{0}\right)^{1 / 2} \approx$ $0.4 \mathrm{fF}$ (see below for our estimation of the effective value $\tilde{C}$ ). We attribute the present form of oscillations to insufficiently high impedance of the biasing resistors. Simple estimations show that the observed upper frequency limit for a single-band behaviour of about $1 \mathrm{GHz}$ is achieved by the system biased only slightly above its Coulomb blockade threshold, therefore resulting in a typical decay time profile of oscillations. A broad spectrum of such oscillations, with considerable contribution of higher harmonics, makes it difficult to ensure their effective phase-locking to an external high-frequency signal. In particular, the preliminary experiments on microwave irradiation of our resistor-biased arrays up to frequencies of about $1 \mathrm{GHz}$ did not result in observable features in the $I V$-curves. On the other hand, it was shown in [11] for the Josephson oscillations that even such oscillations may be effectively phase-locked using a driving signal of an appropriate waveform.

As an indication of a hysteretical behaviour due to the inductance term in the equation of motion (11) [8], [9], the biasing point at small currents $I_{\text {bias }} \sim 10 \mathrm{pA}$ was found to be unstable, exhibiting relatively slow irregular voltage oscillations, see left inset in Fig. 2, As shown schematically in Fig. 3, random switching of the array from the blockade to the finitecurrent state was followed by a relatively slow recharging of the line capacitance $C_{\mathrm{L}}$, which made possible the real-time observation of the switching cycles. In the blockade state, $\mathrm{A} \rightarrow \mathrm{B}$, with a life-time $\Delta t \sim \tau_{\mathrm{D}}$, the current source charged the input terminal of the array until its 
switching, $\mathrm{B} \rightarrow \mathrm{C}$, to the finite-current state, $I_{\text {bias }} \sim 1 \mathrm{nA}$, followed by a rapid discharging, $\mathrm{C} \rightarrow \mathrm{D}$, down to voltages $V_{\mathrm{r}} \approx 500 \mu \mathrm{V}$ and retrapping, $\mathrm{D} \rightarrow \mathrm{A}$, facilitated by the presence of fluctuations (cf. the thermally enhanced retrapping process in a shunted Josephson junction [12]).

Whilst at $T<100 \mathrm{mK}$ the average escape time, $t_{\text {mean }} \equiv\langle\Delta t\rangle$, was nearly temperatureindependent (cf. [13]), as shown in Fig. 4 the switchings at $T>100 \mathrm{mK}$ were due to thermal escape over the barrier whose height we have evaluated to be $\Delta U \approx 70 \mu \mathrm{eV}$, as estimated from the slope of the Ahrrenius plot, see inset to Fig. 4

Using the approach [14] which is valid, strictly speaking, only in the weak-coupling case $\lambda \ll 1$, we obtained a rough estimate of the effective junction capacitance and the length of a soliton in the array. It is possible to express the barrier height $\Delta U(V)$ through the threshold voltage $V_{\mathrm{T}}$ of soliton motion:

$$
\Delta U(V) \approx \frac{e V_{\mathrm{T}}}{2}\left(1-\frac{V}{V_{\mathrm{T}}}\right)^{2} .
$$

Taking advantage of the narrow range of switching voltages at $T>100 \mathrm{mK}, V \approx V_{\mathrm{r}}$, we estimated $V_{\mathrm{T}} \approx 830 \mu \mathrm{V}$, a soliton energy $E \equiv \Delta U(0)=e V_{\mathrm{T}} / 2=e^{2} /\left(2 \tilde{C} C_{0}\right)^{1 / 2} \approx 420 \mu \mathrm{eV}>$ $E_{\mathrm{C}}$, the effective Bloch capacitance $\tilde{C} \sim 1.6 \mathrm{fF} \gg C_{\mathrm{T}}$, and the size of a soliton $M \sim 8$. One can see that the effective Bloch capacitance greatly exceeds the geometric junction capacitance which is obviously due to the strong suppression of the Bloch band width, $\Delta E=E^{\max }(q)-E^{\min }(q)$, for the values of $\lambda>1$ [see, e.g., the limiting case, $\lambda \gg 1$, described by equation (2)]. The shape of a static $2 e$-soliton in the infinitely long array is shown in Fig. [5 as calculated by numerically solving the equation (1) in the stationary case $\partial q_{i} / \partial t \equiv 0$, for several representative values of the ratio $E_{\mathrm{J}} / E_{\mathrm{C}}$.

\section{CONCLUSIONS AND OUTLOOK}

Our dc measurements pointed out the presence of autonomous Bloch oscillations in a resistively biased array of small Josephson junctions. Compared to the experiments with single Bloch junctions reported by other groups, similar Bloch frequencies are expected, whereas the Coulomb voltage threshold and, as a consequence, the amplitudes of oscillations are considerably larger,

making a potential advantage of using the arrays for phase-locking experiments. However, to enable an external synchronization of these oscillations one should realize at least several times 
higher bias impedance, possibly in form of very resistive microstrips. A certain increase in the Bloch frequency could be expected for the junctions with a larger Josephson energy. But since the voltage scale is also affected by the values of $E_{\mathrm{J}}$ and $E_{\mathrm{C}}$, a detailed optimization is still important. Using the statistics of the spontaneous state switchings, we evaluated the effective junction capacitance and the length of a Cooper pair soliton. These data can be helpful for future device development. For practical applications, the influence of the background charges in the islands of the array on the soliton dynamics should also be investigated.

\section{ACKNOWLEDGEMENTS}

The authors would like to thank D. V. Averin and A. V. Ustinov for helpful discussions. Technical assistance from T. Weimann (e-beam lithography), S. A. Bogoslovsky (measurement setup), F.-J. Ahlers and V. A. Rogalya (software) is gratefully acknowledged. The work was partially supported by the EU through the projects RSFQubit and EuroSQIP. 


\section{REFERENCES}

[1] M. W. Keller, "Standards of current and capacitance based on single-electron tunneling devices," in: Recent Advances in Metrology and Fundamental Constants, T. J. Quinn, S. Leschiutta, and P. Tavella Eds., Amsterdam: IOS Press, 2001, pp. 291-316.

[2] M. W. Keller, A. L. Eichenberger, J. M. Martinis, and N. M. Zimmerman, "A capacitance standard based on counting electrons," Science, vol. 285, pp. 1706-1709, 1999.

[3] K. K. Likharev and A. B. Zorin, "Theory of the Bloch-wave oscillations in small Josephson junctions," J. Low Temp. Phys., vol. 59, pp. 347-382, 1985.

[4] L. S. Kuzmin and D. B. Haviland, "Observation of the Bloch oscillations in an ultrasmall Josephson junction," Phys. Rev. Lett., vol. 67, pp. 2890-2893, 1991.

[5] D. B. Haviland, L. S. Kuzmin, P. Delsing, K. K. Likharev, and T. Claeson, "Experimental evidence for the Coulomb blockade of Cooper pair tunneling and Bloch oscillations in single Josephson junctions," Z. Phys. B - Condensed Matter, vol. 85, pp. 339-347, 1991.

[6] L. S. Kuzmin, "Experimental evidence for the autonomous Bloch oscillations in single Josephson junctions," IEEE Trans. Appl. Supercond., vol. 3, pp. 1983-1986, 1993.

[7] D. V. Averin and K. K. Likharev, "Single-electronics: correlated transfer of single electrons and Cooper pairs in small tunnel junctions," in Mesoscopic Phenomena in Solids, B. L. Altshuler, P. A. Lee, and R. A. Webb Eds., Amsterdam: Elsevier, 1991, pp. 175-271.

[8] P. Ågren, K. Andersson, and D. B. Haviland, "Kinetic inductance and Coulomb blockade in one dimensional Josephson junction arrays," J. Low Temp. Phys., vol. 124, pp. 291-304, 2001.

[9] A. B. Zorin, "Bloch inductance in small-capacitance Josephson juncitons," Phys. Rev. Lett., vol. 96, pp. 167001, 2006.

[10] S. V. Lotkhov, S. A. Bogoslovsky, A. B. Zorin, and J. Niemeyer, "Frequency-locked current of Cooper pairs in superconducting single electron transistor with ohmic resistor," in International Workshop on Superconducting NanoElectronics Devices, J. Pekola et al. Eds., New York: Kluwer Academic/Plenum Publishers, 2002, pp. $105-114$.

[11] R. Monaco, "Enhanced ac Josephson effect,” Appl. Phys. Lett., vol. 68, pp. 679-687, 1990.

[12] E. Ben-Jacob, D. J. Bergman, B. J. Matkowsky, and Z. Schuss, "Lifetime of oscillatory steady states," Phys. Rev. A, vol. 26, pp. 2805-2816, 1982.

[13] K. Andersson and D. B. Haviland, "Escape from a zero-current state in a one-dimensional array of Josephson junctions," Phys. Rev. B, vol. 67, pp. 092507, 2003.

[14] N. S. Bakhvalov, G. S. Kazacha, K. K. Likharev, and S. I. Serdykova, "Single-electron solitons in one-dimensional tunnel structures," Sov. Phys. JETP, vol. 68, pp. 581-586, 1989. 


\section{FIGURES}

Fig.1. SEM picture of a fragment of the resistor-biased array of two-junction SQUIDs fabricated with the three-angle evaporation technique.

Fig.2. The $I V$-curves of two slightly different samples. Left inset: a typical time trace of the self-sustaining switching cycles observed at low bias currents. Right inset: a close-up look of the negative-slope segments in the $I V$-curves (shown by horizontal arrows) corresponding to single-band Bloch oscillations.

Fig.3. An equivalent electrical circuit (a) and a cycle diagram in the $I V$-plane (b), both explaining the switching/retrapping process. For the sake of simplicity, the on-chip Cr resistors and the four-point connection layout of the experiment are not shown. The Josephson array is schematically represented by the hatched box. In the presence of fluctuations (external noise, thermal fluctuations, etc.) there is no stable bias point along the load line, which results in the self-sustaining voltage relaxation oscillations observed in experiment.

Fig.4. Mean lifetimes of the blockade state. Inset: Ahrrenius plot at high temperatures.

Fig.5. Calculated shape of a 2e-soliton presented in a form of the island charges $Q_{i}=q_{i+1}-$ $q_{i}=C_{0} V_{i}$, where $q_{i}$ is a quasicharge of an $i$-th junction and $V_{i}$ is a voltage on the $i$-th island. 


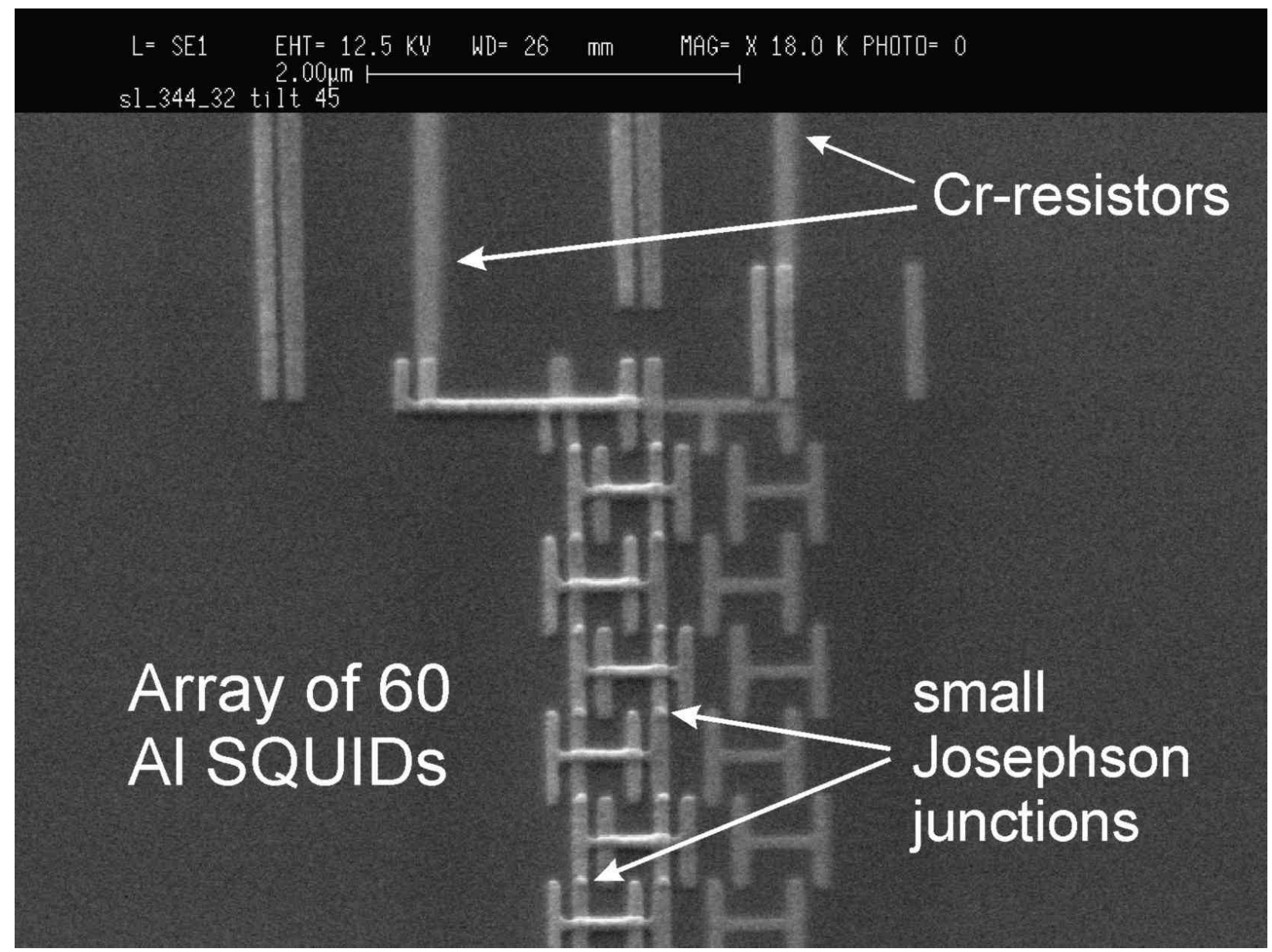

Fig. 1.

S. V. Lotkhov et al. "Cooper pair transport in a resistor-biased Josephson junction array" 


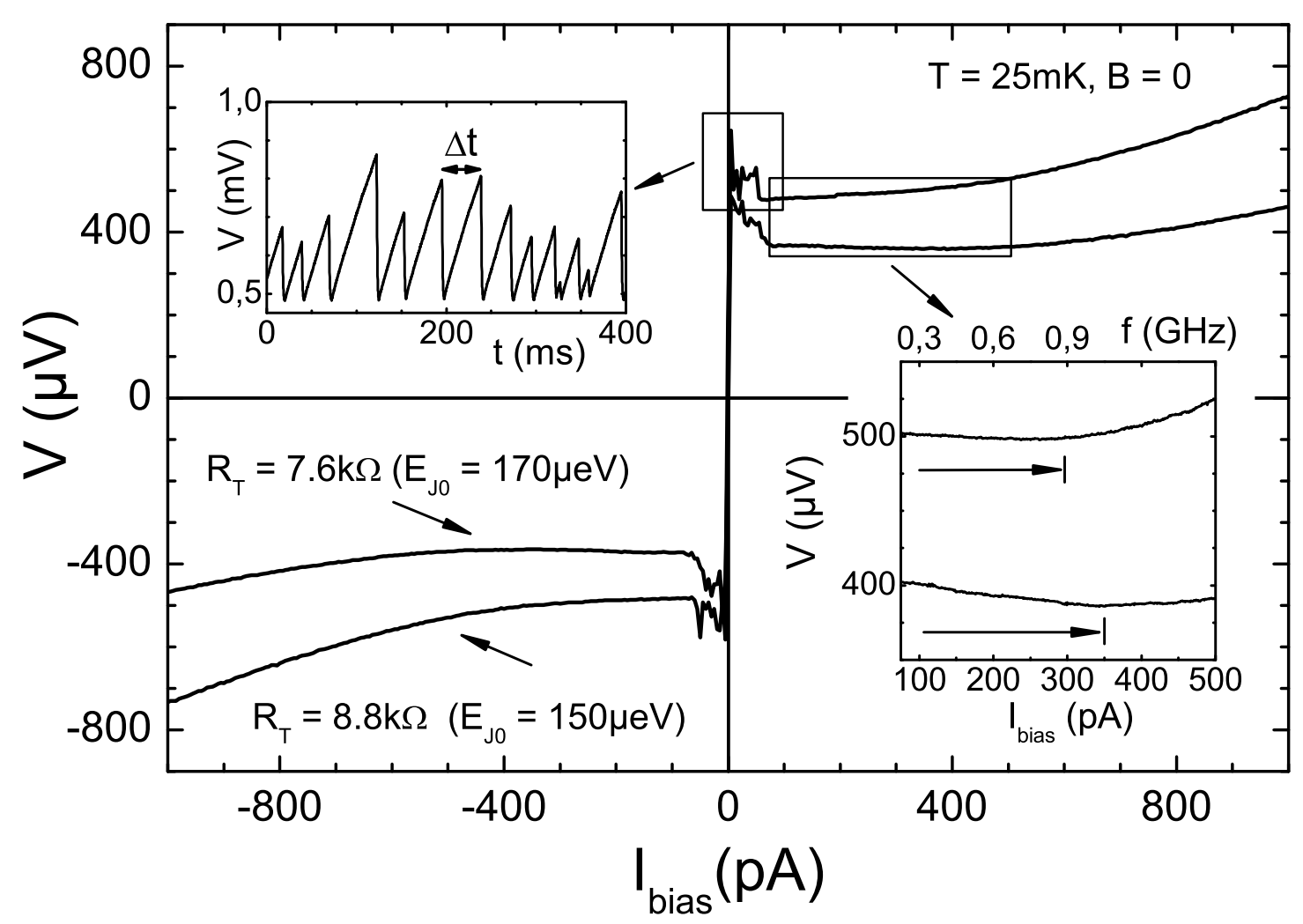

Fig. 2.

S. V. Lotkhov et al. "Cooper pair transport in a resistor-biased Josephson junction array" 
(a)

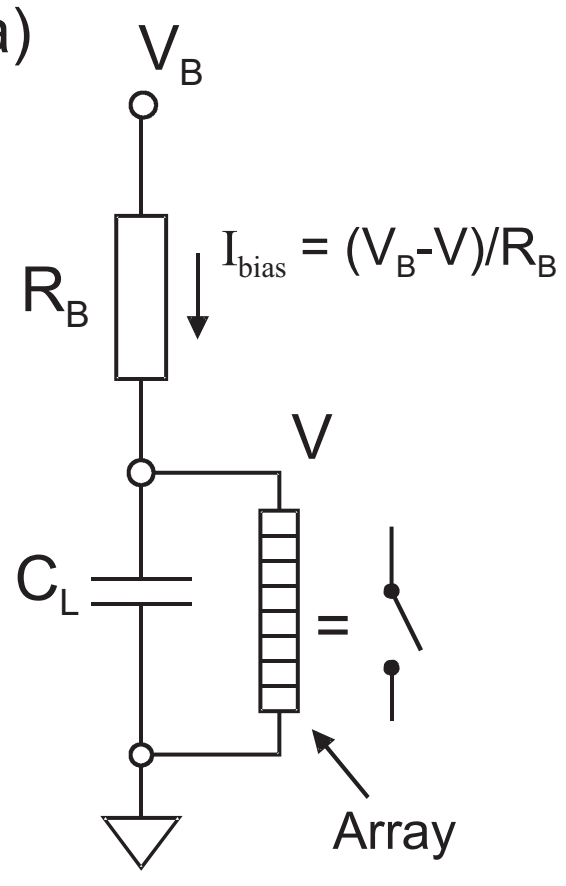

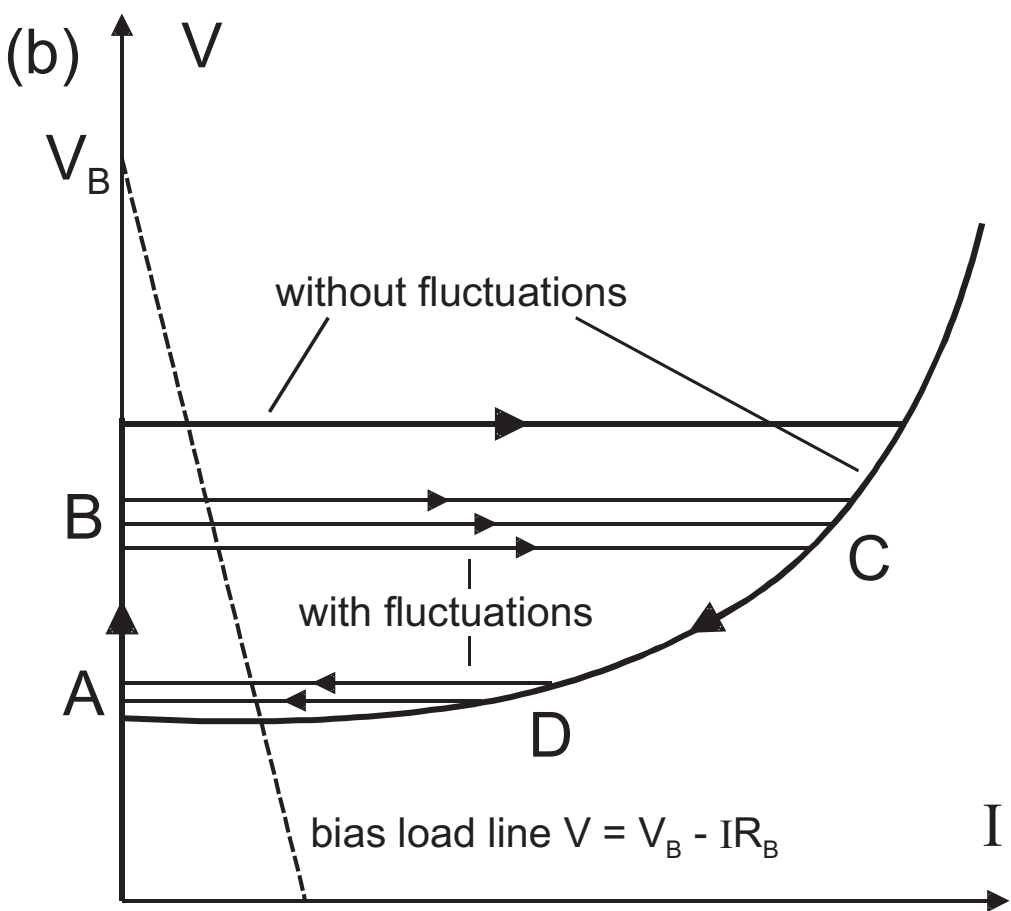

Fig. 3.

S. V. Lotkhov et al. "Cooper pair transport in a resistor-biased Josephson junction array" 


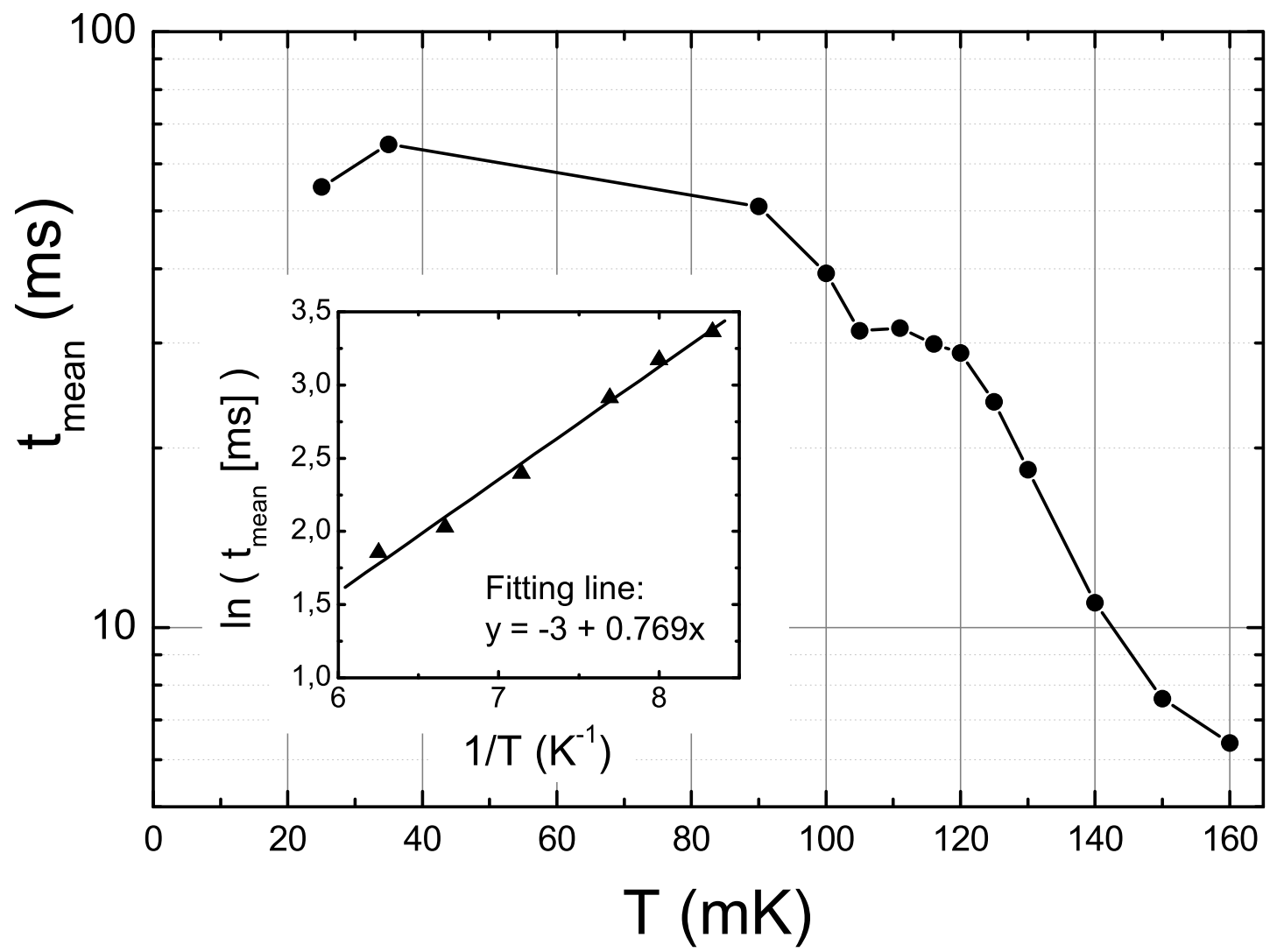

Fig. 4.

S. V. Lotkhov et al. "Cooper pair transport in a resistor-biased Josephson junction array" 


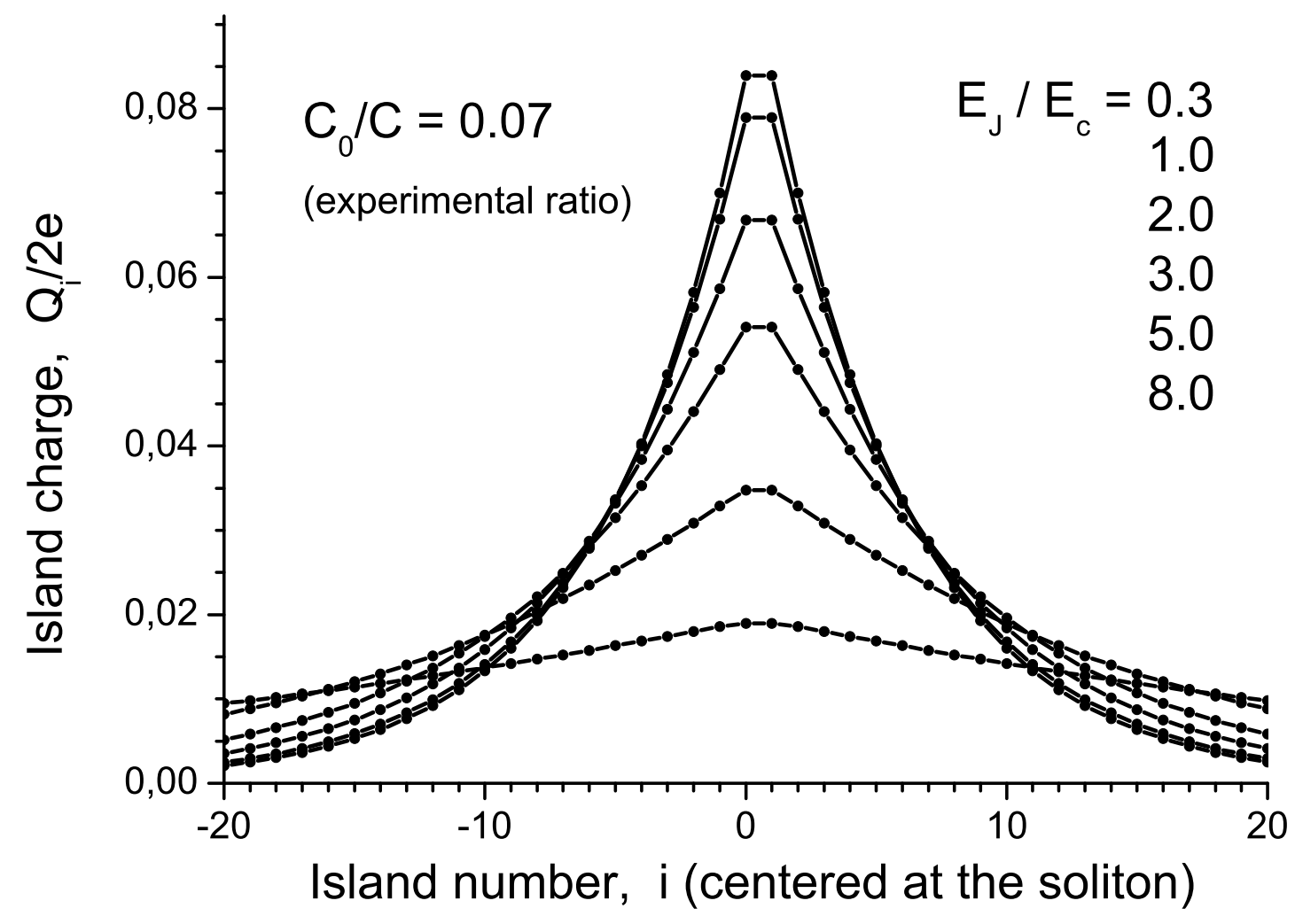

Fig. 5.

S. V. Lotkhov et al. "Cooper pair transport in a resistor-biased Josephson junction array" 